\title{
Equilibrium evaluation for Wendelstein 7-X experiment programs in the first divertor phase
}

\author{
T. Andreeva ${ }^{1 *}$, J. A. Alonso ${ }^{2}$, S. Bozhenkov ${ }^{1}$, C. Brandt ${ }^{1}$, M. Endler ${ }^{1}$, G. Fuchert $^{1}$, J. Geiger ${ }^{1}$, \\ M. Grahl ${ }^{1}$, T. Klinger ${ }^{1}$, M. Krychowiak ${ }^{1}$, A. Langenberg ${ }^{1}$, S. Lazerson ${ }^{3}$, U. Neuner ${ }^{1}$, \\ K. Rahbarnia ${ }^{1}$, N. Pablant ${ }^{3}$, A. Pavone ${ }^{1}$, J. Schilling ${ }^{1}$, J. Schmitt ${ }^{4}$, H. Thomsen ${ }^{1}$, Y. Turkin ${ }^{1}$ \\ and the W7-X Team \\ ${ }^{1}$ Max-Planck-Institute for Plasma Physics, Wendelsteinstraße 1, 17491 Greifswald, Germany \\ ${ }^{2}$ Laboratorio Nacional de Fusión, CIEMAT, Av. Complutense 40, 28040 Madrid, Spain \\ ${ }^{3}$ Princeton Plasma Physics Laboratory, Princeton NJ, 08543, USA \\ ${ }^{4}$ Auburn University, Department of Physics, Auburn AL, 36849, USA
}

Wendelstein 7-X (W7-X) is a modular advanced stellarator, which successfully went into operation in December 2015 at the Max-Planck-Institut für Plasmaphysik in Greifswald, Germany, and continued to thrive at the experimental campaign with the first divertor phase in August-December 2017. The nested magnetic surfaces in W7-X are created by a system of 3-D toroidally discrete coils, providing both toroidal and poloidal field components, and designed with the aim to create optimum equilibrium properties. The optimization criteria included the high quality of vacuum magnetic surfaces, good finite beta equilibrium and MHD-stability properties as well as a substantial reduction of the neoclassical transport and bootstrap current in comparison to classical stellarators.

Equilibrium calculations, devoted to the analysis of the experiment programs dedicated to measure the bootstrap current, were performed with help of the Variational Moments Equilibrium Code, available as Wendelstein 7-X web service. Pressure profiles based on experimental data served as an input for calculations. The mapping of measurements using precalculated equilibria for different beta values has been analyzed. A comparison with reconstruction results obtained by means of the Minerva Framework, considering only experimental data of magnetics measurements, and the V3FIT code is presented.

Keywords: Wendelstein 7-X, first divertor phase, equilibrium, reconstruction, stellarator, OP1.2a.

\section{Introduction}

The mission of the Wendelstein-X project (W7-X) is to demonstrate the reactor potential of the optimized stellarator line. Besides the high quality of vacuum surfaces as a prerequisite for a good confinement, good finite- $\beta$ equilibrium and MHD stability properties in order to ensure high fusion power, low neoclassical transport in the $1 / v$ regime for a large confinement time, good collisionless containment of fast-ions necessary for fusion $\alpha$-particles, steady state operation and feasible modular coil design, a small bootstrap current is one of the principal optimization criteria [1]. The minimization of the bootstrap current is essential to preserve the W7-X edge structure [2] and ensure the safe divertor operation. For example, a toroidal current of $10 \mathrm{kA}$ causes a radial shift of the islands of approximately $2 \mathrm{~cm}$, and can shift the divertor strike lines even more in comparison with their designed positions:

$$
\begin{gathered}
\Delta t=\frac{\Delta B_{\theta} \cdot R}{B_{\varphi} \cdot r}=\frac{\mu_{0} I_{\text {tor }} \cdot R}{2 \pi r^{2} \cdot B_{\varphi}} \approx 2 \cdot 10^{-3} I_{\text {tor }}[\mathrm{kA}] \\
\Delta r[\mathrm{~cm}]=\frac{\Delta t}{d_{t} / d r} \approx 0.2 \cdot I_{\text {tor }}[\mathrm{kA}]
\end{gathered}
$$

In (1) $\dot{i}$ is the rotational transform, $B_{\ominus}$ - poloidal magnetic field, $\mathrm{R}-$ major radius, $\mathrm{r}-$ minor radius, $\mathrm{B}_{\varphi}-$ toroidal magnetic field, $\mathrm{I}_{\text {tor }}$ - toroidal current.

The verification of the bootstrap current minimization was one of the milestones in the experimental campaign with the first divertor phase (OP1.2a), conducted in August-December 2017. For this purpose, long (more than 10 seconds) discharges were performed in three main reference magnetic configurations, which were examined during this campaign. Figure 1 shows the time evolution of the measured toroidal current in two discharges in the standard (EIM) configuration, in one discharge in the high mirror (KKM) configuration and in one discharge in the high iota configuration (FTM) [3].

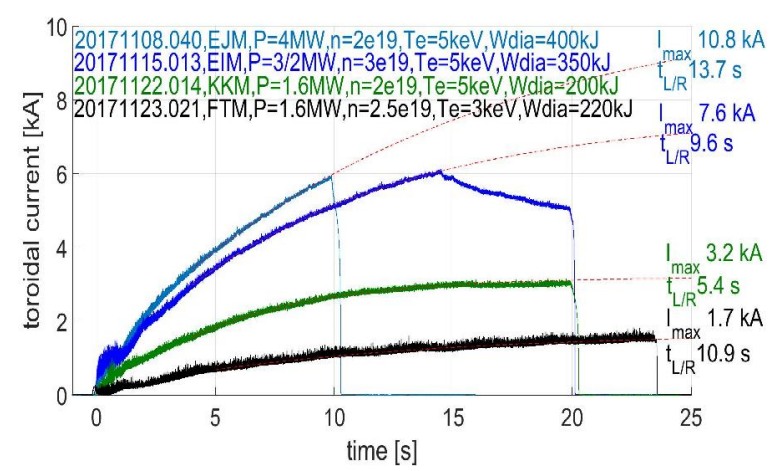

Fig. 1. Time evolution of toroidal currents measured in OP1.2a. Blue lines correspond to two discharges with slightly different experiment parameters in the standard magnetic configuration, green line corresponds to the high mirror magnetic configuration, black line - to the high iota magnetic configuration. Red dashed lines show the individual configuration fits, estimating the stationary bootstrap current value. The $L / R$ time is the output parameter of the fits. 
Necessary for the equilibrium simulations radial plasma profiles of electron density and electron temperature were measured by means of the Thomson Scattering system [4], the ion temperature profile - with the high resolution Xray imaging spectrometer (XICS) [5] and $Z_{\text {eff }}$ values were measured spectroscopically (line integrated measurement assuming a constant value over the profile). The toroidal current was measured by means of Rogowski coils [6, 7]. For the chosen plasma parameters the value of the toroidal current is almost negligible in the high iota configuration and reaches its highest values in the standard configuration, being characterized by the maximum value of approximately $10 \mathrm{kA}$. The observed configuration dependency follows the theoretical optimization expectations. For the further evaluations, the discharge with the highest measured bootstrap current XP_20171108.040 (standard configuration) was chosen, since the impact on plasma equilibrium will be the largest.

\section{Mapping of the diagnostic data and equilibrium evaluation}

The first preparative step for the equilibrium modelling was a mapping of the experimental density and temperature profiles in order to obtain the pressure profiles necessary for the VMEC [8] runs. The standard quick procedure to map diagnostic data is to use the precalculated equilibria available via $\mathrm{W} 7-\mathrm{X}$ web services [9]. In the web services data collection, the pre-calculated equilibria can be found for all W7-X main reference configurations for different theoretical pressure profiles and different beta values. In the first experimental campaign phase OP1.2a with a divertor, the main part of the discharges was characterized by rather low beta values, and typically $\beta=0 \%$ equilibrium calculations were taken for a mapping.

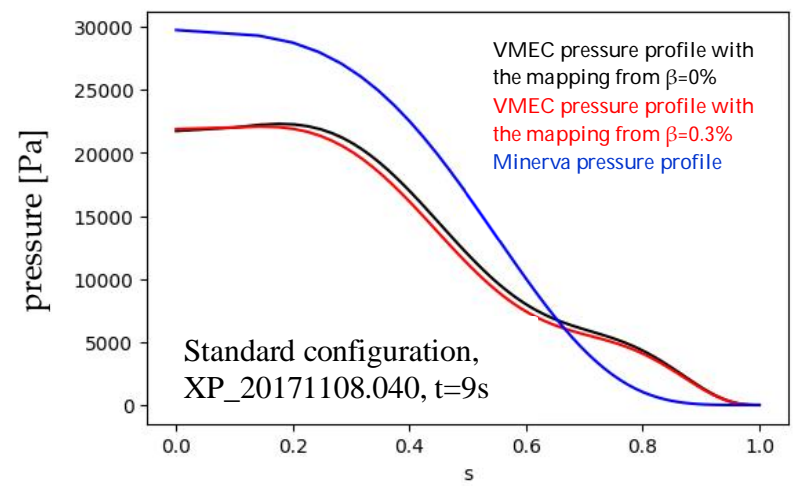

Fig. 2. VMEC pressure profiles obtained from the mapping with the $\langle\beta\rangle=0 \%$ (black line) and $\langle\beta\rangle=0.3 \%$ (red line) pre-calculated equilibria. Blue line corresponds to the pressure profile used in Minerva calculations. Horizontal axis represents the radial coordinate $s=\psi_{\text {tor }} / \psi_{\text {LCMS }}$ (normalized toroidal magnetic flux).

To check the validity of this approach for the analysis of the bootstrap current discharges (previous section) two VMEC calculations were performed for the discharge XP_20171108.040, $t=9 \mathrm{~s}$ with the electron density and electron temperature profiles mapped from the $\langle\beta\rangle=0 \%$ and $\langle\beta\rangle=0.3 \% \quad$ (corresponding to the estimated experiment values) pre-calculated equilibria.
Figure 2 shows the comparison of the VMEC pressure profiles obtained from the mapping with the $\langle\beta\rangle=0 \%$ (black curve) and $\langle\beta\rangle=0.3 \%$ (red curve) pre-calculated equilibria. The difference between both profiles appears to be insignificant, and the same holds for the VMECcalculated values of the rotational transform (Fig. 3, red and black lines, almost coinciding with each other). The kinetic energy values found in these two calculations were $346 \mathrm{~kJ}$ and $330 \mathrm{~kJ}$ respectively, while the diamagnetic energy measured in the discharge was found to be $355 \mathrm{~kJ}$ [10]. These results justify the usage of $\beta=0 \%$ mapping for the low beta OP1.2a discharges.

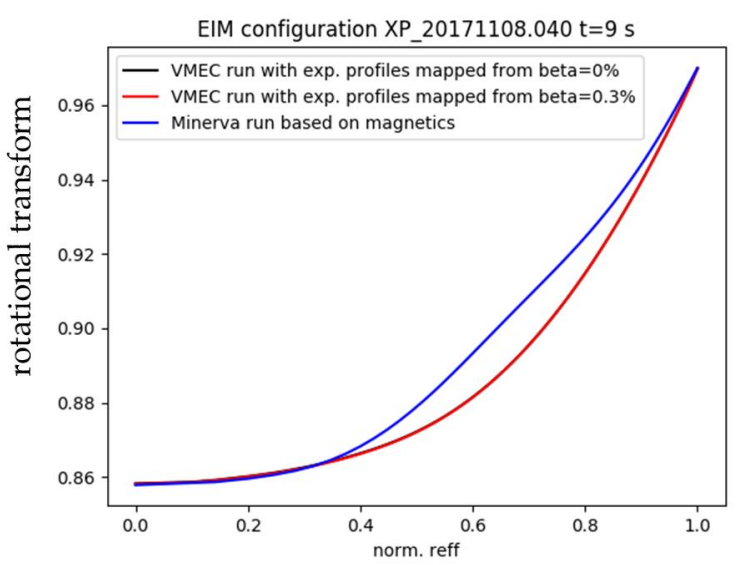

Fig. 3. Rotational transform, obtained for the bootstrap current discharge XP_20171108.040, $\mathrm{t}=9 \mathrm{~s}$ from VMEC calculations with the experimental pressure profile mapped from precalculated equilibria, with $\langle\beta\rangle=0 \%$ (black line), from precalculated equilibria with $\langle\beta\rangle=0.3 \%$, (red line) and Minerva framework analysis (blue line).

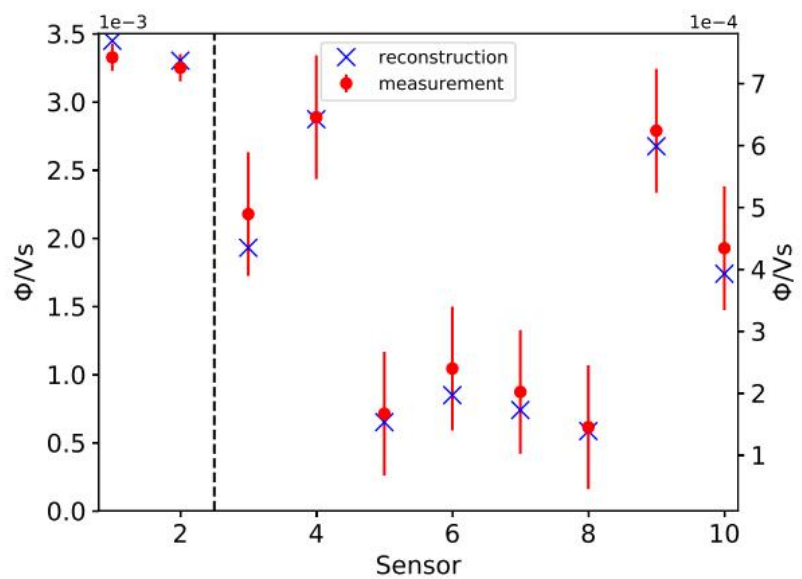

Fig. 4. Minerva evaluation of the XP_20171108.040 [12]. Blue crosses show simulated values of magnetic signals corresponding to the diamagnetic loop (1), the continuous Rogowski coil (2) and eight sensors of segmented Rogowski coils (3)-(10), red dots - to their measured values.

The equilibrium calculations performed with the VMEC code based on experimental profiles and $\mathrm{Z}_{\mathrm{eff}}$ measurements were compared with the Bayesian modeling framework Minerva [11] analysis taking into account only a limited set of magnetic measurements and no profile information for the equilibrium evaluation. 
The Minerva framework is a large-scale analysis tool using Bayesian inference to build a complex relationship between physical models and diagnostic measurements. A reconstruction of the MHD equilibrium using the Minerva framework for the analysis of the discharge XP_20171108.040 employed a magnetic diagnostics prediction code using signals of the diamagnetic loop, one continuous Rogowski coil and eight segmented Rogowski coil signals (Fig. 4). The blue line in Figure 3 shows the obtained rotational transform profile, which only slightly deviates from the VMEC calculations, which are based on experimental data of electron and ion temperatures, electron density and $Z_{\text {eff }}$ measurements. The small difference in the shape of the rotational transform profile is most likely caused by the differences in pressure profiles used in VMEC and Minerva calculations (Fig. 2). Figure 5 illustrates the Shafranov shift obtained for this discharge, which is of order $1 \mathrm{~cm}$ [12].

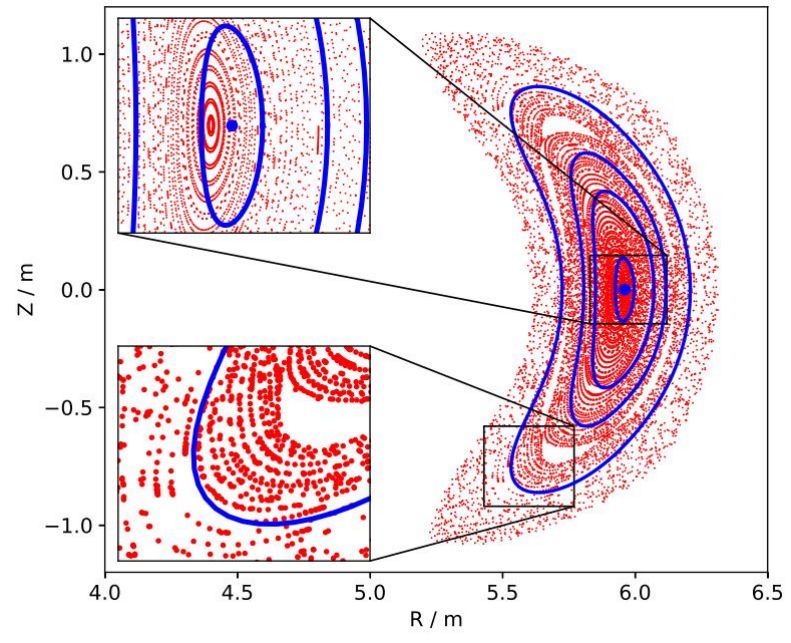

Fig. 5. Shafranov shift obtained for the XP_20171108.040 discharge. Red dots correspond to Poincare plots in the vacuum case, blue line - to Minerva output for XP_20171108.040.

We find that for low beta discharges the Minerva reconstruction delivers reliable results even with a limited part of the magnetic diagnostic signals and no profile data as input.

\section{Comparison with V3FIT reconstruction results.}

The V3FIT equilibrium reconstruction code [13] utilizes several diagnostic signals, including magnetics, Thomson scattering, interferometry, and geometric divertor information, in a non-linear optimization algorithm with singular-value decomposition to rapidly calculate the equilibrium state of 3-D magnetically confined plasmas. It has been used to reconstruct current and pressure profiles in the quasi-helically symmetric stellarator HSX $[14,15]$, as well as current and density profiles in the compact toroidal hybrid experiment [16]. V3FIT is used on $\mathrm{W} 7-\mathrm{X}$ to reconstruct radial profiles of the plasma current, and the plasma pressure, along with profiles of the electron temperature and density as well as (argon) impurity ion temperatures from XICS.

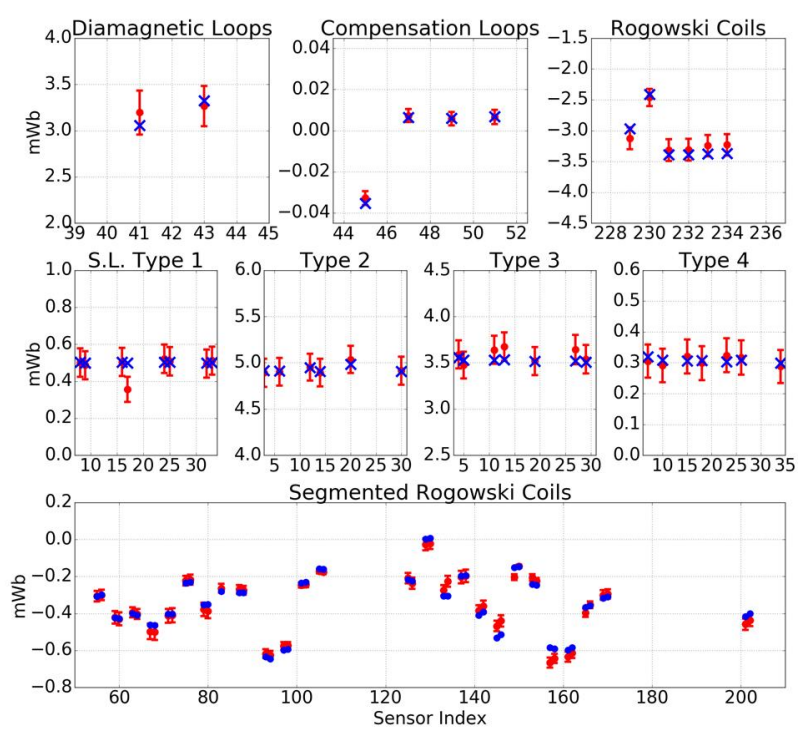

Fig. 6. Input magnetic diagnostics signals used for the V3FIT reconstruction: red - measured magnetic signals with error bars, blue - simulated values. The units for all diagnostics are milliWebers.

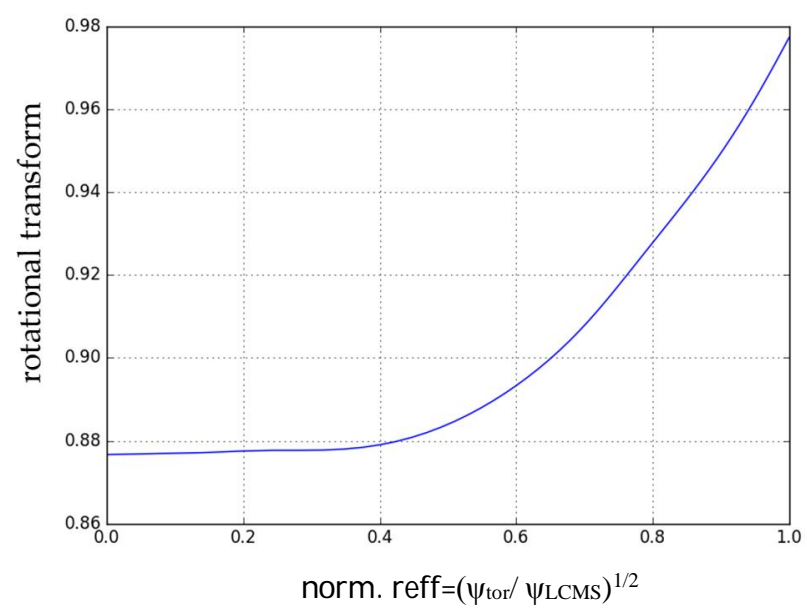

Fig. 7. Rotational transform profile obtained from the V3FIT reconstruction for XP_20171108.040.

Table 1. Comparison of the diamagnetic energy Edia measured by the diamagnetic loop in the XP_20171108.040 and kinetic energy $E_{k i n}$ obtained in different equilibrium reconstruction simulations. (1) - VMEC run, based on experimental pressure profiles mapped using the pre-calculated equilibria with $\beta=0 \%$, (2) - VMEC run, based on experimental pressure profiles mapped from the pre-calculated equilibria with $\beta=0.3 \%$, (3) Minerva Framework analysis based on magnetic signals only, (4) - V3FIT reconstruction, based on magnetic signals only.

\begin{tabular}{|l|l|l|l|l|}
\hline & $(1)$ & $(2)$ & $(3)$ & $(4)$ \\
\hline $\mathrm{E}_{\text {kin }}[\mathrm{kJ}]$ & 346 & 330 & 364 & 363 \\
\hline Diff. to $\mathrm{E}_{\text {dia }}[\%]$ & 2.5 & 7 & 2.5 & 2.3 \\
\hline
\end{tabular}

To reconstruct the XP_20171108.040 discharge only magnetic diagnostics signals served as an input, which was similar to the Minerva reconstruction, but in the V3FIT reconstruction a larger number of magnetic diagnostic signals was used. Figure 6 shows the measured magnetic signals (assuming approximately 5\% 
uncertainty) in red with related error bars. The corresponding modeled signals, based on the reconstructed profiles, are indicated as blue crosses. The plots in the top row correspond to the diamagnetic loops, compensation loops and continuous Rogowski coils. The second row corresponds to the four types of saddle coils and the bottom row shows the segmented Rogowski coils. Figure 7 represents the rotational transform profile obtained from this V3FIT reconstruction. The corresponding output value of the kinetic energy was 363 $\mathrm{kJ}$.

Table 1 represents a comparative overview of the obtained results with respect to the kinetic energy simulation for the discharge XP_20171108.040 performed by VMEC modelling, based on experimental pressure profiles, Minerva Framework analysis using only a limited set of magnetic signals and V3FIT reconstruction, also with magnetic signals alone as an input. All reconstruction methods show a difference to the value of the diamagnetic energy measured in the discharge of less than $7 \%$, which indicates an acceptable agreement.

\section{Conclusions}

Bootstrap current measurements were performed in the first divertor operation phase OP1.2a of W7-X for the three reference magnetic configurations examined in this campaign and qualitatively confirmed theoretical predictions on $\mathrm{W} 7-\mathrm{X}$ optimization.

The W7-X web service equilibrium data collection covers all main reference configurations and provides a reliable basis for diagnostic mapping as well as for data analysis.

OP1.2a experimental profiles related to the bootstrap current discharges can be reliably mapped with $\beta=0 \%$ precalculated equilibria.

VMEC equilibrium evaluation based on OP1.2a measured pressure profiles shows good agreement with Minerva Framework equilibrium reconstruction based only on magnetic diagnostic data and with the V3FIT reconstruction analysis.

Further equilibrium analysis of W7-X magnetic configurations can be performed on the basis of Minerva Framework as well as the V3FIT and STELLOPT reconstruction codes [17].

\section{Acknowledgments}

This work has been carried out within the framework of the EUROfusion Consortium and has received funding from the Euratom research and training programme 20142018 under grant agreement number 633053. The views and opinions expressed herein do not necessarily reflect those of the European Commission.

\section{References}

[1] G. Grieger, et al., "Modular stellarator reactors and plans for Wendelstein 7-X", Fusion Technol. 21 (1992) 1767-1778.
[2] J.D.Lore, et al., "Design and analysis of divertor scraper elements for the W7-X stellarator," IEEE Trans. Plasma Sci., vol. 42, no. 3, (2014) 539-544

[3] T. Andreeva, et al., "Characteristics of main configurations of Wendelstein 7-X", Problems of Atomic Science and Technology, Series: Plasma Physics, Vol. 4, (2002) 45-47.

[4] S. Bozhenkov, et al., "The Thomson scattering diagnostic at Wendelstein 7-X and its performance in the first operation phase", Journal of Instrumentation, Volume 12 (2017) 10004.

[5] N.A. Pablant, A. Langenberg, et al. "Core Radial Electric Field and Transport in Wendelstein 7-X Plasmas", Physics of Plasmas, 25, 022508 (2018).

[6] K. Rahbarnia, et al., "Comissioning of the magnetic diagnostics during the first operation phase at Wendelstein 7-X", Proc. 43rd EPS Conference, Leuven, Belgium (2016).

[7] M. Endler, et al., "Engineering design for the magnetic diagnostics of Wendelstein 7-X", Fusion Engineering and Design, 100 (2015), 468-494.

[8] S. Hirshman, W. van Rij, P. Merkel, "Three-dimensional free boundary calculations using a spectral Green's function method", Comput. Phys. Comm., 43 (1986), 143-155.

[9] M. Grahl, et al. "Web services for 3D MHD equilibrium data at Wendelstein 7-X", IEEE Transactions on Plasma Science, Volume 46, Issue 5 (May 2018) 1114 - 1119.

[10] K. Rahbarnia, et al., "Diamagnetic energy measurement during the first operational phase at the Wendelstein $7-\mathrm{X}$ stellarator" Nuclear Fusion, Volume 58, Number 9 (2018) 096010 (9pp).

[11] J. Svensson and A. Werner, "Large scale Bayesian data analysis for Nuclear Fusion experiments", in 2007 IEEE International Symposium on Intelligent Signal Process., Conference Proceedings Book (2007), pp. 955-960.

[12] J. Schilling, "Experimental MHD Equilibrium Analysis of Magnetic Configurations in the Wendelstein 7-X Stellarator", MSc thesis, techn. rep., IPP 2018-20.

[13] J.D. Hanson, D.T. Anderson, M. Cianciosa, et al., "Nonaxisymmetric equilibrium reconstruction for stellarators, reversed field pinches and tokamaks", Nucl. Fusion 53083016 (2013).

[14] J.C. Schmitt, et al, "Measurement of a helical PfirschSchlüter current with reduced magnitude in HSX", Nucl. Fusion 53, 0820001 (2013).

[15] J.C. Schmitt, J.N. Talmadge, D.T. Anderson and J.D. Hanson, "Modeling, measurement and 3-D equilibrium reconstruction of the bootstrap current in the Helically Symmetric Experiment”, Phys. Plasmas 21, 092518 (2014).

[16] M. R. Cianciosa, J. D. Hanson, D. A. Maurer, "Uncertainty Analysis in 3D Equilibrium Reconstruction", Fusion Science and Technology, 74:1-2, 1-12 (2018).

[17] S. Lazerson and the DIII-D Team "Three-dimensional equilibrium reconstruction on the DIII-D device", Nuclear Fusion, 55 (02), 023009 (2015). 